\title{
Impacto dos Gastos Públicos com Educação e Saúde no Crescimento Econômico dos Estados Brasileiros ${ }^{1}$
}

\section{Impact of Public Spending on Education and Health on Economic Growth in the Brazilian States}

Francisco José Silva Tabosa ${ }^{1}$

Pablo Urano de Carvalho Castelar ${ }^{2}$

\begin{abstract}
Resumo
O presente trabalho tem como objetivo medir o impacto dos gastos públicos nas áreas de educação e saúde no crescimento econômico dos estados brasileiros, para o período de 1991 a 2012, período escolhido pela disponibilidade de dados e que apresenta o parâmetro da revisão de literatura abordada. Utilizaram-se três métodos distintos para captar essa análise: primeiro utilizando efeitos fixos e aleatórios, segundamente fez-se uso das técnicas econométricas de regressões quantílicas para dados em painel e, por último, o Método dos Momentos Generalizados, desenvolvido por Blundell-Bond (1998). Os resultados mostraram que, para todas as três metodologias utilizadas no estudo, tanto os gastos com educação, quanto os gastos com saúde impactam positivamente, mesmo que de forma tímida, no crescimento econômico.
\end{abstract}

Palavras-Chave: Crescimento Econômico. Brasil. GMM. Regressão Quantílica. Gastos Públicos.

\begin{abstract}
This study aims to measure the impact of public spending in the areas of education and health on the economic growth of Brazilian states, for the period 1991 to 2012, a period chosen by the availability of data and which presents the parameter of the literature review addressed. Three different methods were used to capture this analysis: first, using fixed and random effects, second, using econometric techniques of quantile regressions for panel data, and, finally, the Generalized Moments Method, developed by Blundell-Bond (1998). The results showed that for all three methodologies used in the study, both spendings on education and on health had, albeit timidly, a positive impact on economic growth.
\end{abstract}

Keywords: Economic Growth. Brazil. GMM. Quantile Regression. Public Spending.

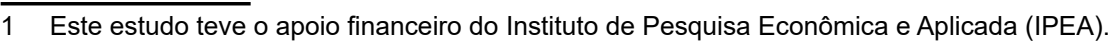

Economista. Dr. Professor do Programa de Pós Graduação em Economia Rural da Universidade Federal do Ceará (PPGER/UFC). Bolsista produtividade do CNPq. 


\section{INTRODUÇÃO}

A preocupação com os gastos públicos na economia é recorrente, especialmente em relação aos impactos desses no crescimento econômico. Tal relação, entre gasto público e crescimento econômico, tem atraído o interesse de diversos pesquisadores (Vogt et al., 2015; Abu-Bader e Abu-Qarn, 2003; Rodrigues e Teixeira, 2010; Barro, 1991; Barro e Sala-i-Martin, 1995). Nesse sentido, a hipótese geralmente formulada e que encontra respaldo na evidência sugere que os gastos públicos podem elevar o crescimento econômico, aumentando assim a produtividade do setor privado.

Como ressaltam, por exemplo, Rodrigues e Teixeira (2010), a relevância do governo no desenvolvimento é devida à sua capacidade de incentivar direta e indiretamente a acumulação dos determinantes de crescimento econômico, onde atividades como a acumulação de capital físico são influenciadas pelos gastos governamentais com formação bruta de capital fixo. A teoria do crescimento endógeno, como exposta em Barro e Sala-i-Martin (1995), ressalta que existem ainda externalidades relativas à oferta de bens públicos e semipúblicos que elevam os retornos privados dos investimentos, as taxas de poupança e a própria acumulação de capital. O próprio trabalho de Rodrigues e Teixeira (2010), que analisa o impacto dos gastos públicos nas esferas municipal, estadual e federal, no crescimento econômico do Brasil, apresenta evidência nesse sentido, principalmente o impacto dos gastos em nível de estado.

Assim, o objetivo do presente trabalho é analisar o impacto dos gastos públicos com educação e saúde no crescimento econômico. O intento é averiguar se, para o caso brasileiro, observa-se a corroboração do resultado observado em extensa literatura empírica, que encontra relação positiva entre gastos em saúde e educação com crescimento econômico ou aumento de renda.

Como argumentado por Marques Jr. et al. (2006), os gastos públicos podem ser considerados produtivos ou improdutivos; improdutivo quando o setor público investe recursos em áreas que competem com o setor privado e estes não geram benefícios para a sociedade. Ressalte-se, também, que autores como Srinivasan (1985) e Buchanan (1980) argumentam que gastos públicos podem ser improdutivos e não geram produto adicional, sendo apenas a extração de renda, ou rent-seeking, resultante da disputa entre diferentes grupos de interesse que exercem pressão por benefícios ou subsídios.

Já o caso produtivo ocorre quando o gasto passa a ser introduzido de forma positiva na função de produção local e diretamente útil aos consumidores, gerando benefícios (Vogt et al., 2015).

Keynes (1936) argumenta que existe uma relação entre o orçamento do governo e a atividade econômica de um país, visto que corte nos gastos governamentais reduziriam o consumo e o produto. Contudo, Summers e Heston (1988), numa estimação para 130 países, analisaram os gastos com defesa e educação, e concluíram que esse tipo de gasto, de forma geral, parece ser improdutivo. Devarajan et al. (1996), usando dados de 43 países num período de 20 anos, observam que gastos com capital, saúde, educação, transporte e comunicação são considerados normalmente produtivos, mas podem ser improdutivos, caso sejam realizados em excesso. Baldacci et al. (2008) analisaram 118 países em desenvolvimento, de 1971 a 2000, e concluíram que os gastos com educação e saúde apresentam um impacto positivo sobre o crescimento econômico.

Como discutido em Rocha e Giuberti (2007), para realizar a avaliação do impacto do gasto público no crescimento econômico, existem duas possibilidades; ou se utiliza uma matriz de insumo-produto - sendo assim uma avaliação indireta -, ou por meio de análises estatísticas. Dessa forma, o presente estudo busca analisar essa relação utilizando a segunda opção, que tenta avaliar a ligação entre gasto público e crescimento econômico nos estados brasileiros.

Especificamente, o foco do estudo é em gastos com educação e saúde, visto que representam os dois fatores preponderantes no crescimento econômico (Pirim, Owings e Kaplan, 2014; Campodonico, Cassinelli e Mesones, 2014; Barro, 1991; Barro, 1990). Além disso, utilizou-se o superávit (déficit) primário como um fator explicativo do crescimento econômico. A argumentação que motiva incluir essa última variável está presente, por exemplo, em Gerardo (2010), onde, estudando a política fiscal e sustentabilidade do crescimento no Brasil, ressalta que "a importância da política fiscal no Brasil decorre do seu papel como mecanismo de coordenação das demais políticas e sua inter-relação com as demais variáveis de política econômica. Assim, as decisões dos agentes econômicos quanto ao nível e ao financiamento do déficit público têm reflexos sobre a taxa de juros e a inflação. Essas variáveis, em um processo de retroalimentação, afetam a dívida pública influenciando as expectativas dos agentes econômicos com relação à sustentabilidade da dívida e o esforço fiscal requerido para evitar trajetória explosiva do estoque da dívida" (GERARDO, 2010, p. 10). Desta forma, uma política fiscal que almeja um superávit primário tenderia a favorecer o crescimento econômico num momento posterior.

Em termos metodológicos, utilizaram-se três métodos distintos para captar essa análise. O intento dessa abordagem é trabalhar com dados em painel, onde se mesclam dados transversais com séries de tempo. Os dados transversais são os estados da federação, e a série temporal de 1991 a 2012. Com isso, através desses métodos, é possível avaliar os estados de maneira comparativa, assim como realizar uma avaliação ao longo do tempo.

O primeiro método consistiu em utilizar o modelo econométrico, para dados em painel com efeitos fixos e aleatórios. O seguinte método fez uso das técnicas econométricas de regressões quantílicas para dados em painel. Por último, foi utilizado o Método dos Momentos Generalizados desenvolvido por Blundell-Bond (1998). 
O estudo é composto com cinco seções além desta introdução (seção 1). A seção 2 apresentará uma revisão de literatura de estudos internacionais e nacionais sobre a relação gastos com educação e saúde e crescimento econômico. A seção 3 abordará a metodologia empregada no estudo. Na seção 4 apresentaremos os resultados obtidos. E por fim, com as conclusões (seção 5) se encerra o estudo.

\section{REVISÃO DE LITERATURA}

A presente seção tem como objetivo compilar algumas das principais contribuições acadêmicas que, de alguma maneira, têm compatibilidade com o tema da relação entre o gasto público e o crescimento econômico. Os trabalhos são apresentados por temas, iniciando da relação geral dos gastos públicos com crescimento econômicos, e, posteriormente, comentando pesquisas específicas, que focam na relação do crescimento com a educação, com a saúde, e, por fim, na relação dos gastos públicos em educação e saúde com o crescimento.

Um dos estudos pioneiros da relação gasto público e crescimento econômico, principalmente o crescimento endógeno, foi o estudo de Barro (1990). Para o autor, gastos com a defesa e educação são considerados produtivos para o crescimento econômico. Aschauer (1989) concluiu, de modo similar, que os gastos públicos com crescimento econômico provocam elevações do produto. Contudo, esses gastos eram centralizados em infraestrutura.

Em tempos mais recentes, Ventelou e Bry (2006), utilizando o método da Análise Envoltória de Dados (DEA) para analisar a relação entre gastos públicos e crescimento econômico nos países da Organização para a Cooperação e Desenvolvimento Econômico (OCDE), descobrem, primeiramente, que há indícios de que o gasto público modifica o potencial para o crescimento macroeconômico. Além disso, os resultados do artigo sugerem que o impacto do gasto público no crescimento aparenta ser maior quando a análise envoltória de dados é utilizada como pré-condição para a abordagem econométrica e, por fim, advogam pela utilização de um índice de bem-estar, dado que pontos aquém da fronteira de eficiência não são meras instâncias de erro de alocação de recursos.

A literatura brasileira também tem analisado o tema, com algumas contribuições relevantes. Sant'Anna (2006), por exemplo, analisou a relação Produto Interno Bruto (PIB) com os gastos sociais (saúde e saneamento, educação, assistência e previdência) nos municípios do estado do Espírito Santo, mas não verificou uma relação estatística significante.

Rocha e Giuberti (2007) investigam a relação gasto público em educação, saúde, transporte, comunicação e defesa com o crescimento econômico dos estados brasileiros entre os anos de 1986 a 2003. Os autores verificaram que os gastos com educação apresentaram um efeito positivo em relação ao crescimento econômico.

Já Silva (2012) buscou analisar o impacto de diversas formas de gasto público com o crescimento econômico nos municípios paraibanos. Os resultados mostraram que os gastos com educação tiveram baixa elasticidade-produto, influenciando de forma tímida o crescimento econômico na região.

Algumas pesquisas focam especificamente no tema educação e sua relação com o crescimento econômico ou aumento da renda. Glomm e Ravikumar (1992), por exemplo, analisaram o impacto do nível de escolaridade das famílias no aumento do nível de renda. Os autores concluíram que maiores gastos do governo em educação, principalmente no nível superior, proporcionam maiores níveis de renda.

Já em relação à saúde, diversas contribuições especificamente para esse setor também podem ser discutidas. Grossman (1972) enfatiza que regiões em que a população se encontra com condições da saúde precárias (saneamento básico, higiene etc., proporcionando assim uma maior vulnerabilidade a doenças) afetam negativamente o crescimento econômico, via redução da produtividade da mão de obra no setor privado com o aumento de licenças saúde, internações, baixo nível de habilidade etc.

Nessa mesma linha de raciocínio, Lillard e Weiss (1997) estabelecem uma ligação entre saúde e renda futura, onde um impacto positivo das condições de saúde no desempenho educacional das famílias, aumentando a produtividade e consequentemente o nível de renda ou riqueza das famílias.

Bloom e Canning (2000) descrevem uma relação entre saúde e crescimento econômico, enfatizando que melhores condições de saúde das famílias proporcionam maior nível de energia física e mental, aumentando assim a produtividade da mão de obra, além de um maior aprendizado em termos de educação. Assim, afetaria positivamente o crescimento econômico de um país.

Aurangzeb (2001) investigou a relação gastos com saúde e crescimento econômico no Paquistão, no período de 1973 a 2003. Utilizando o modelo de crescimento de Solow (1956) e testes de cointegração de Johansen e modelo de Correção de Erros (VEC), o autor concluiu que existe uma relação positiva entre os gastos com saúde e crescimento econômico, tanto no curto quanto em longo prazo. Bloom et al. (2004), utilizando a técnica de mínimos quadrados em dois estágios e uma amostra de dados de vários países, verificaram uma relação positiva entre expectativa de vida e escolaridade com o crescimento econômico.

Outro estudo relevante foi de Lustig (2006), que analisou a relação saúde (taxa de mortalidade e outros indicadores de saúde, como taxa de internação da população economicamente ativa, número de contaminações, etc.) e crescimento econômico no México, no período de 1970 a 1995. Como resultado, a autora também encontra uma relação positiva entre saúde e crescimento econômico. 
Suhrcke et al. (2006) utilizam dados de taxa de mortalidade adulta, taxa de fertilidade e expectativa de vida para analisar o crescimento econômico na Estônia. O estudo mostrou uma relação negativa entre as variáveis taxa de fertilidade e de mortalidade adulta com o crescimento econômico. Além disso, os autores verificam que a saúde tinha uma relação direta com a oferta de trabalho e o nível de produtividade dos trabalhadores.

Oni (2014) e Bakare e Sanmi (2011) analisaram o impacto dos gastos com saúde no crescimento econômico da Nigéria. Como indicadores de saúde, foram utilizados taxa de mortalidade, taxa de casos de AIDS ${ }^{2}$ na fase adulta, número de internações na fase adulta. Os autores verificaram que esses gastos proporcionaram uma maior produtividade da mão de obra, impactando positivamente no crescimento econômico no país. Ainda no referido país, Ayuba (2014) analisa os gastos com educação e saúde no crescimento econômico, no período de 1990 a 2009. Fazendo uso do modelo de correção de erros, os resultados afirmaram a relação positiva entre os gastos e o crescimento econômico em longo prazo.

Boussalem, Boussalem e Taiba (2014) analisaram a relação entre gastos com saúde e crescimento econômico na Argélia durante o período de 1974 a 2014. Utilizando testes de causalidade e cointegração, os autores verificaram que existe uma relação positiva, mas que o crescimento econômico é que proporciona o aumento dos gastos com saúde. A evidência empírica sugere, então, que a relação inversa não ocorre.

Contudo, Devlin e Hansen (2001) encontram uma causalidade bidimensional entre gastos com saúde e crescimento econômico, em uma amostra de 15 países da OCDE de elevada renda per capita, para o período de 1995-2011. Da mesma forma, Cole e Neumayer (2006) tentam verificar se uma condição precária de saúde (má nutrição e doenças como malária) afeta o desenvolvimento econômico via produtividade total dos fatores, e encontram que os baixos níveis de saúde da população podem reduzir a produtividade, podendo ser um motivo para o não desenvolvimento em muitas regiões no mundo.

Kambiz Peykarjou et al. (2011) analisaram os gastos públicos e crescimento econômico nos países membros da OIC (Organization Islamic Conference), no período de 2001 a 2009. Outros fatores além dos gastos com saúde foram analisados, como expectativa de vida e taxa de fertilidade. Os resultados mostraram uma relação positiva entre gastos com saúde e crescimento econômico, mas uma relação negativa entre taxa de fertilidade e crescimento econômico.

Bedir (2016) analisou a relação crescimento econômico e gastos com saúde em alguns países da União Européia, Oriente Médio e Àsia, no período de 1995 a 2013. Fazendo uso de testes de causalidade de Granger (modificada por Toda e Amamoto (1995) apud Bedir (2016)), verificaram que o crescimento econômico é um fator importante para diferentes tipos de gastos com saúde nos países.

Em outros estudos aplicados no Brasil, e mais recentemente em municípios, a maioria dos trabalhos procura mensurar a eficiência de hospitais, escolas. Nesta linha de pesquisa de gastos municipais encontramos Marinho (2001), Bezerra e Diwan (2001) e Faria (2005). Este último estudo abordou os gastos municipais do estado do Rio de Janeiro, onde os dados utilizados referem-se ao ano de 2002, com exceção do indicador de resultado para educação que mede o desempenho dos alunos em exame de proficiência referente ao ano de 2004, com múltiplos insumos e múltiplos produtos. Já o trabalho desenvolvido por Brunet et al. (2006) analisou os gastos públicos estaduais no Brasil por funções do orçamento, embora tenha utilizado Free Disposal Hull (FDH).

Barbosa e Souza (2015) analisaram, através da Análise Envoltória de dados (DEA), a eficiência técnica e escala do Índice de Desempenho do Sistema Único de Saúde (SUS) dos municípios do nordeste brasileiro. Os resultados indicam que os municípios nordestinos com maiores índices referentes às especificidades apresentaram, em média, os melhores escores de eficiência técnica e de escala do Índice de Desempenho do Sistema Único de Saúde (IDSUS) nos municípios do Nordeste brasileiro.

Por fim, temos uma diversidade de trabalhos voltados a estudar a relação de interesse da presente pesquisa, i.e., a relação da educação e da saúde no crescimento econômico.

Santos (2008), por exemplo, analisou os gastos com capital humano (anos de estudo e gastos com saúde) no estado do Ceará, e seu impacto no crescimento econômico nos municípios cearenses. O resultado mostrou uma relação positiva entre as variáveis. O PIB dos municípios se mostrou ser pouco sensível, inelástico e influenciado positivamente por gastos com saúde e saneamento (Araújo, Moraes e Monteiro, 2013).

Maita e Mukhopadhyay (2012) estudam a relação entre os gastos públicos com educação e saúde e o crescimento econômico em 12 países na Ásia e no Pacífico. Em seis países ${ }^{3}$, verificaram-se uma relação positiva entre as variáveis analisadas, através do teste de cointegração de Johansen. Já em outros países ${ }^{4}$, essa relação não foi verificada. Nas Filipinas, no entanto, foi apresentada uma relação negativa entre gastos com educação e crescimento econômico. Já os gastos com saúde impactaram positivamente o crescimento econômico em Blangladesh, Nepal, Filipinas, Singapura e Sri Lanka.

Alshahrani e Alsadiq (2014) examinaram os efeitos dos gastos públicos com educação e saúde no crescimento econômico na Arábia Saudita, no período de 1969 a 2010. Utilizando um modelo de crescimento de Ram (1986), modelos de Vetores Autorregressivos (VAR) e modelo de Correção de Erros (VEC), os autores verificaram que os

\footnotetext{
2 A Nigéria está entre os países com maior nível de contaminação de casos de AIDS. Mais detalhes ver Oni (2014)

3 Bangladesh, Kiribati, Malásia, Maldivas, Filipinas e República da Corea.

4 Fiji, Nepal, Singapura, Sri Lanka, Tongo e Vanuatu.
} 
gastos com saúde e educação só apresentaram efeitos positivos no crescimento econômico em longo prazo. Já em curto prazo esse resultado não se verifica.

Lacheheb, Nor e Baloch (2014) examinaram a relação entre os gastos públicos com educação e saúde e crescimento econômico nos países do MENA ${ }^{5}$. Usando técnicas econométricas para dados em painel, e uma base de dados de 20 países membros, no período de 1995 a 2010, os autores encontram um efeito positivos entre os gastos com o crescimento econômico na região. Por exemplo, se os gastos com saúde e educação aumentarem $100 \%$, proporcionam um aumento no PIB real em torno de $27,2 \%$ e $34,7 \%$, respectivamente.

Campodonico, Cassinelli e Mesones (2014) analisaram a relação entre os gastos com saúde, educação e infraestrutura com o crescimento econômico no Peru, durante o período de 1973 a 2011. Os resultados mostram que, em longo prazo, os gastos conseguiram promover o crescimento econômico no país, reduzindo assim o desemprego, pobreza e desigualdade de renda. Contudo, os gastos com educação e saúde tiveram um impacto menor do que os gastos com infraestrutura.

Pirim, Owings e Kaplan (2014) estudam os efeitos de longo prazo dos gastos com saúde e educação na taxa de desemprego em 50 estados americanos, durante 25 anos. Os resultados sugerem que o mecanismo mais eficiente para a redução do desemprego foram os gastos com educação. Contudo, os gastos com saúde também apresentaram um efeito positivo na redução do desemprego.

De maneira similar, Eggoh, Houeninvo e Sossou (2015) analisaram a relação entre capital humano (gastos com educação e saúde) e crescimento econômico em 49 países africanos, no período de 1996 a 2010. Utilizando o Método Econométrico de Painel Dinâmico, os autores encontraram que os gastos com educação e saúde tiveram um impacto negativo no crescimento econômico na região. No entanto, os indicadores de capital humano, como anos de estudo, por exemplo, tiveram impacto positivo. Tal fato possivelmente reflete que os investimentos realizados pelos governos africanos foram, de uma forma geral, improdutivos, não proporcionando crescimento econômico na região.

Churchill, Yew e Ugur (2015) buscaram verificar os efeitos dos gastos com educação e saúde com o crescimento econômico nos países membros do $\mathrm{OCDE}^{6}$, no período de 1980 a 2000. Os resultados obtidos mostraram um efeito positivo entre os gastos com educação e crescimento econômico. Todavia, em relação aos gastos com saúde, esse efeito teve um sinal negativo, mas estatisticamente insignificante.

Por fim, Tarda e Rodrigues (2015) analisaram os gastos com educação e crescimento econômico na região administrativa de Campinas/SP para o ano de 2007; utilizando a analise econométrica dos Mínimos Quadrados Ordinários, onde os autores concluíram que os gastos com educação possuem um impacto positivo no crescimento econômico da região.

Vagt, Degenhart e Zonatto (2015) analisaram os gastos públicos com educação e saúde, bem como sua relação com o crescimento econômico nos municípios da região sudeste do Brasil. Os dados foram coletados para o ano 2010, através do Censo Demográfico, que constituiu os dez maiores e menores municípios de cada estado da região, além de mostrar uma relação positiva entre os gastos e o crescimento econômico na região. Os autores salientam que os gastos públicos apresentam ter um papel importante para o crescimento econômico na região. Por fim, resultado semelhante foi obtido por Bogoni, Hein e Beuren (2011), mas nesse caso foi utilizada a mesma técnica para os estados da região sul.

\section{METODOLOGIA}

\subsection{Base de Dados}

A base de dados foi composta por informações de 27 Unidades da Federação (UF), para o período de 1991 a 2012. O período escolhido, além de levar em consideração a disponibilidade de dados que contemplem todas as variáveis, busca se adequar, em grosso modo, ao período de análise da maioria das pesquisas mencionadas na revisão de literatura.

Todas as informações foram obtidas na Secretaria do Tesouro Nacional (STN) e IPEADATA. Ressalta-se que todas as variáveis monetárias foram atualizadas para valores reais de 2012, utilizando como deflator o Índice Nacional de Preços ao Consumidor (INPC), tendo como base o período de outubro de 2012.

As variáveis utilizadas nesse estudo foram:

Txcrescpib = Taxa de crescimento real do Produto Interno Bruto das Unidades federativas do Brasil;

Gastedu = Percentual dos gastos públicos estaduais com educação em relação ao seu respectivo Produto Interno Bruto;

Gastsaud = Percentual dos gastos públicos estaduais com saúde em relação ao seu respectivo Produto Interno Bruto;

5 Países do Oriente Médio e Norte da Africa.

6 Organização para a Cooperação e Desenvolvimento Econômico, com 37 países. 
SDP = Superávit (ou déficit) Primário, obtido através da diferença entre as receitas correntes e despesas correntes das Unidades Federativas, em proporção ao Produto Interno Bruto ${ }^{7}$;

TC = Taxa de cambio;

TXDES = Taxa de desemprego nas Unidades federativas.

A Tabela 1 apresenta a estatística descritiva de todas as variáveis do estudo. Observa-se que, em média, a taxa de crescimento real dos estados (Txcrescpib), no período de 1991 a 2012, foi de 2,15\% ao ano. Os gastos com educação (Gastedu) e saúde (Gastsaud) foram em média 2,64\% e 2,19\% dos seus PIB's estaduais, respectivamente. Os estados obtiveram, em média, superávits primários, representando cerca de $2 \%$ dos seus respectivos PIB's estaduais. A taxa de desemprego foi, em média, $8,52 \%$ ao longo do período analisado.

Tabela 1 - Estatística descritiva das variáveis utilizadas no estudo

\begin{tabular}{cccccc}
\hline Variáveis & Observações & Média & Desvio padrão & Mínimo & Máximo \\
\hline Txcrescpib & 594 & 0.0215 & 0.3841 & -0.0557 & 0.1279 \\
Gastedu & 594 & 0.0264 & 0.0219 & 0.0019 & 0.1387 \\
Gastsaud & 594 & 0.0219 & 0.0219 & 0.0017 & 0.1033 \\
SDP & 594 & 0.0196 & 0.0221 & -0.1100 & 0.1092 \\
TC & 594 & 1.6153 & 0.8964 & 0.001 & 3.0783 \\
TXDES & 594 & 8.52 & 2.7446 & 2.7099 & 20.5395 \\
\hline
\end{tabular}

Fonte: Secretaria do Tesouro Nacional e IPEADATA. Elaboração dos autores.

\subsection{Aspectos metodológicos}

Nessa seção são apresentadas as metodologias empregadas nesse estudo buscando verificar o impacto dos gastos públicos no crescimento econômico dos estados brasileiros, no período de 1991 a 2012.

A primeira delas foi o método de dados em painel com efeitos fixos e aleatórios, fazendo uso do Teste de Hausman para identificar qual o modelo mais apropriado. Em seguida, utilizou-se o método de regressões quantílicas para dados em painel. Por fim, utilizou-se o Método dos Momentos Generalizados (GMM) desenvolvido por Blundell-Bond (1998).

\subsubsection{Modelos fixos e aleatórios}

O modelo econométrico a ser estimado foi:

(1)

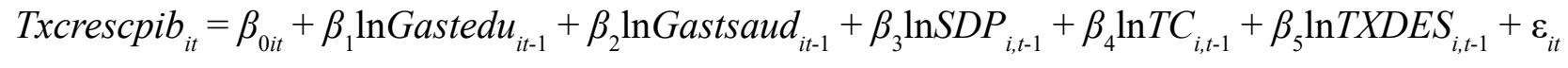

Onde:

Txcrescpibj $_{i, t}=$ Taxa de crescimento real do Produto Interno Bruto do estado $i$ no período $t$;

In Gasted $_{i, t-1}=$ Logaritmo natural dos gastos com educação em proporção ao Produto Interno Bruto do estado $i$ no período t-1;

In Gastsaud $_{i, t-1}=$ Logaritmo natural dos gastos com saúde em proporção ao Produto Interno Bruto do estado $i$ no período $t-1$;

$\mathbf{S D P}_{i, t-1}=$ Logaritmo natural do superávit (ou déficit) primário do estado $i$ no período $t-1$;

$\mathbf{T C}_{i, t-1}=$ Logaritmo natural da taxa de câmbio no período $t-1$;

TXDES $_{i, t-1}=$ Logaritmo natural da taxa de desemprego do estado $i$ no período $t-1$;

$i=$ Estados $(1, \ldots, 27)$

$\boldsymbol{t}=$ Períodos $(1991, \ldots, 2012)$.

Observa-se que o termo correspondente aos efeitos individuais $\left(\beta_{0}\right)$ mantém-se constante ao longo do tempo $(t)$, sendo, talvez, preferível tratá-lo de forma aleatória e não determinística, a fim de se destacar a heterogeneidade individual de cada curso. Sendo assim, o vetor de "constantes" do modelo (1) passa a ser considerado como aleatório: $\beta_{0 i, t}=\beta_{01, t}+\mu_{i}$ em que $E\left(\mu_{i}\right)=0$.

7 Nessa diferença, se a variável apresentar sinal positivo, compreende-se que as receitas correntes foram maiores do que as despesas, ocasionando um déficit fiscal. Todavia, se a variável apresentar sinal positivo, compreende-se que as receitas correntes foram superiores as despesas correntes, provocando assim um superávit fiscal. 
De acordo com Greene (2000), a escolha de uma ou de outra especificação (fixa ou aleatória) deve ser procurada nos pressupostos comportamentais da base de dados. No entanto, de acordo com a literatura especializada, a utilização do teste de especificação de Hausman faz-se necessária. Vale salientar, que a utilização de uma especificação incorreta poderá conduzir a problemas de especificação ou consistência dos estimadores (GREENE, op. cit.). O teste de especificação está definido na equação (2).

(2)

$$
H=\left(\beta_{E A}-\beta_{E F}\right)^{\prime}\left(\Sigma_{E F}-\Sigma_{E A}\right)^{-1}\left(\beta_{E A}-\beta_{E F}\right)
$$

Sendo que: $\beta_{E A}$ corresponde aos estimadores de efeitos aleatórios; $\beta_{E F}$ aos estimadores de efeitos fixos, e; $\left(\Sigma_{E F}-\Sigma_{E A}\right)$ à matriz composta pela diferença entre as variâncias dos estimadores de efeitos fixos e aleatórios.

A estatística do teste terá, sob a hipótese nula da ortogonalidade entre os efeitos individuais aleatórios e regressores, ou seja, de que o estimador de efeitos aleatórios é o apropriado, uma distribuição assintótica $\chi^{2}$ com $K$ graus de liberdade. Caso a hipótese nula não possa ser aceita, o modelo de efeitos fixos é o que apresenta os melhores estimadores, enquanto que os estimadores do modelo de efeitos aleatórios continuam consistentes, mas não podem ser considerados eficientes.

\subsubsection{Modelo de regressões quantílicas para dados em painel}

A análise econométrica foi realizada utilizando regressões quantílicas que apresenta algumas vantagens sobre o modelo de Mínimos Quadrados Ordinários (MQO). Primeiro, as estimações na média de MQO tendem a dar um peso excessivo a observações discrepantes na explicação da variável dependente. Todavia, a regressão quantílica lida melhor com observações discrepantes, fornecendo estimativas mais robustas.

Segundo, as estimações de MQO assumem que o termo de erro possui a mesma distribuição, independente das variáveis explicativas, o que nem sempre ocorre. Um modelo de MQO permite apenas a mudança de local na distribuição, enquanto a regressão quantílica permite alterações na curtose e na dispersão, pois não especifica nenhuma distribuição para o erro a priori (OLIVEIRA e LIMA, 2010).

Terceiro, o método de regressão quantílica permite estimar toda a distribuição condicional da variável dependente, uma vez que permite estimar os parâmetros em um intervalo contínuo entre zero e um. Contudo, esta metodologia permite calcular o impacto das variáveis explicativas sobre a variável dependente ao longo da distribuição condicional desta última, enquanto a estimação por MQO nos fornece apenas o efeito dos regressores na média condicional da variável dependente.

A regressão quantílica é uma generalização da regressão condicionada à mediana. Neste caso, a predição da função de regressão é condicionada a um quantil, $\tau$, da variável dependente, por exemplo, o primeiro quartil $(\tau=$ $0,25)$ ou ao nono decil $(\tau=0,90)$.

O procedimento de estimação, de acordo com Penna e Trompieri Neto (2009), assume que a variável dependente (Taxa de Crescimento Real do Produto Interno Bruto - Txcrescpib) da equação (1), $d$, seja uma variável aleatória com função de distribuição de probabilidade tal que $F(d)=\operatorname{Prob}(D \leq d)$. Deste modo, para $0<\tau<1$, o -ésimo quantil de $D$ pode ser definido como o menor $d$ que satisfaça $F(d) \geq \tau$, ou seja, $Q(\tau)=\inf \{d: F(d) \geq \tau\}$.

Assim sendo, dado um conjunto de $n$ observações em $D$, a função de distribuição empírica de $d$ deve ser dada por

(3)

$$
F n(d)=\sum_{k} 1(z)\left(D_{i} \leq d\right)
$$

Onde $1(z)$ é uma função indicadora que toma o valor de 1 caso o argumento $z$ seja verdadeiro e 0 caso contrário. Nestes termos, o quantil empírico associado a esta distribuição será dado por:

(4)

$$
\operatorname{Qn}(\tau)=\inf \{d: F n(d) \geq \tau\}
$$

A equação (4) se resume ao seguinte problema de otimização:

(5)

$$
\text { Qn }(\tau) \arg \min =\left\{\sum_{i=Y_{i} \geq \xi}\left|D_{i}-\xi\right|+\sum_{i=Y_{i} \geq \xi}(1-\tau)\left|D_{i}-\xi\right|\right\}=\arg \min _{\xi}\left\{\sum_{i} \rho_{t}\left|D_{i}-\xi\right|\right\}
$$


Onde $\rho(u)=u(\tau-1(u<0)) \tau$ é conhecida como função check e que valora pesos positivos e negativos assimetricamente.

A regressão quantílica estende esta formulação de modo a permitir regressores $X$. Assume-se uma especificação linear para o quantil condicional da variável $D$, dados valores para um p-vetor de variáveis explanatórias $X$, de modo que:

(6)

$Q\left(\tau \mid X_{i}^{\prime} \beta(\tau)=X_{i}^{\prime} \beta(\tau)\right\}$

Onde $\beta(\tau)$ é o vetor de coeficientes associado ao $\tau$-ésimo quantil.

Assim sendo, o estimador condicionado ao quantil pode ser encontrado de maneira similar ao problema de otimização descrito em (5), ou seja, será dado por:

$\beta n(\tau)=\underset{\beta(\tau)}{\arg \min }\left\{\sum_{i} \rho_{t}\left(D_{i}-X_{i}^{\prime} \beta(\tau)\right\}\right.$

A vantagem deste método é a sua robustez à presença de "outliers" e a possibilidade de se testar o impacto dos gastos públicos condicionados a diferentes quantis da função de taxa de crescimento real dos PIB's estaduais.

\subsubsection{Método dos Momentos Generalizados (GMM)}

Para quantificar o impacto dos gastos públicos no crescimento econômico dos estados brasileiros, utilizou-se também um modelo dinâmico para dados em painel, descrito por:

(8)

$$
\begin{aligned}
& \text { Txcrescpib }_{i, t}=\beta_{0}+\beta_{1} \text { Txcrescpib }_{i t-1}+\beta_{2} \ln \text { Gasted }_{i, t-1}+\beta_{3} \ln \text { Gastsaud }_{i, t-1}+\beta_{4} \ln S D P_{i, t-1}+\beta_{5} \ln T C_{i, t-1}+ \\
& \beta_{6} \ln \operatorname{TXDESS}_{i, t-1}+v_{t}+u_{i, t}
\end{aligned}
$$

Note que o modelo é especificado em logaritmo com o intuito de obter as elasticidades gastas com educação e saúde no crescimento econômico, representadas pelos coeficientes $\beta_{2}$ e $\beta_{3}$, respectivamente.

A especificação do modelo (8) é baseada na suposição de que o crescimento econômico tende a influenciar a dinâmica do crescimento econômico no próximo período. Por isso, se justifica a presença da variável dependente defasada em um período (Txcrescpib ${ }_{i t-1}$ ) como variável explicativa.

As hipóteses do modelo são que a $E\left[v_{1}\right]=E\left[u_{i, t}\right]=E\left[v_{i} u_{i, t}\right]=0$ para $i=1, \ldots, N$ e $t=1, \ldots, T$; o erro não é correlacionado temporalmente, ou seja, $E\left[u_{i, T} u_{i, S}\right]=0$ para $i=1, \ldots, N$ e $\forall t \neq \mathrm{s}$. Além disso, impõe-se a condição inicial de que $E\left[P_{k, i t} \mathrm{u}_{i, t}\right]$ $=0$ para $i=1, \ldots, N$ e $t=1, \ldots, T$ (Ahn e Schimdt, 1995).

A presença de Txcrescpib $_{i t-1}$ como variável explicativa na equação (8) pode levar a problema de endogeneidade, gerando um viés no painel dinâmico. Logo, as estimativas de MQO tendem a ser enviesadas e inconsistentes, superestimando o coeficiente da variável supracitada (Greene, 2000). Uma tentativa para correção desse viés pode ser pelo uso do estimador de efeito fixo Within-groups que gera desvios padrões dos coeficientes ligeiramente menores. Entretanto, para solucionar o problema de endogeneidade, deve-se aplicar a transformação do modelo em primeira diferença e proceder à estimação pelo Método dos Momentos Generalizados (GMM), a partir da minimização das condições de momentos da distribuição. Deste modo, a equação (9) se transforma em:

\section{(9)}

$$
\begin{aligned}
& \Delta \text { Txcrescpib }_{i t}=\beta_{1} \Delta \text { Txcrescpib }_{i t-1}+\beta_{2} \Delta \ln \text { Gasted }_{i, t-1}+\beta_{3} \Delta \ln \text { Gastsaud }_{i, t-1}+\beta_{4} \Delta \ln S D P_{i t-1}+ \\
& \beta_{5} \Delta \ln T C_{i, t-1}+\beta_{6} \Delta \ln T X D E S_{i, t-1}+\Delta u_{i, t}
\end{aligned}
$$

Onde $\Delta$ é um operador de diferenças e, pela construção da equação (9), $\Delta T_{x c r e s c p i b}{ }_{i t-l}$ e $\Delta u_{i, t}$ são correlacionados se a endogeneidade ainda persiste. Logo, utiliza-se algum instrumento para $\Delta$ Txcrescpib $_{i t-1}$. O conjunto de hipóteses adotadas na equação (8) implica que as condições de momentos, $E\left[\Delta\right.$ Txcrescpib $\left._{i t-s} \Delta u_{i, t}\right]=0$ para $t=3,4, \ldots, T$ e $s \geq 2$ são válidas. De acordo com esses momentos, Arellano e Bond (1991) sugerem empregar $\Delta T_{x c r e s c p i b_{i t-s}}$, para $t=3$, $4, \ldots, \boldsymbol{T}$ e $s \geq 2$ como instrumentos para equação (9).

8 Caso em que as variáveis explicativas do modelo são correlacionadas com os resíduos $E\left(Z_{i t}, \varepsilon_{i t}\right) \neq 0$. Uma variável qualquer pode ser classificada como: estritamente exógena, se não é correlacionada com os termos de erro passados, presente e futuros; fracamente exógena, se é correlacionada apenas com valores passados do termo de erro; e endógena, se é correlacionada com os termos de erro passados, presente e futuros. 
O segundo caso, os valores da variável defasada em um ou mais períodos podem ser considerados como instrumentos válidos para estimar a equação (9). Já no último caso, os valores defasados em dois ou mais períodos são instrumentos válidos na estimação da equação (8).

No entanto, Arellano e Bover (1995) e Blundell e Bond (1998) explicam que esses instrumentos são fracos quando as variáveis, dependentes e explicativas, apresentam forte persistência e/ou a variância relativa dos efeitos fixos aumenta. Neste caso, o estimador é inconsistente e enviesado. Como solução para este problema, os autores recomendam a estimação de um sistema que combine o conjunto de equações em diferenças, equação (9), com o conjunto de equações em nível, equação (8), conhecido como método GMM para sistemas (System-GMM).

Para as equações em diferenças, o conjunto de instrumentos é o mesmo descrito acima, enquanto que para regressão em nível, os instrumentos apropriados são as diferenças defasadas das respectivas variáveis. Por exemplo, assumindo que as diferenças das variáveis explicativas não são correlacionadas com os efeitos fixos individuais (para $t=3,4, \ldots, T)$ e $E\left[\Delta\right.$ Txcrescpib $\left._{k, i 2} \nu_{i}\right]=0$ para $i=1,2,3, \ldots, N$, então as variáveis explicativas em diferenças e $\Delta T T_{c r e s c p i b} b_{i-1}$, caso elas sejam exógenas ou fracamente exógenas, são instrumentos válidos para equação em nível. O mesmo se dá se estas são endógenas, mas com os instrumentos sendo as variáveis explicativas em diferenças defasadas de um período e mais $\Delta$ Txcrescpib $_{i t-1}$.

A consistência do estimador System-GMM depende da ausência de correlação serial no termo de erro e da validade dos instrumentos adicionais. Logo, inicialmente, testam-se as hipóteses nulas de ausência de autocorrelação de primeira e segunda ordem dos resíduos. Para que os estimadores dos parâmetros sejam consistentes, a hipótese de ausência de autocorrelação de primeira ordem deve ser rejeitada e a de segunda ordem aceita. Posteriormente, realizaram-se os Testes de Hansen e de Sargan para verificar a validade dos instrumentos utilizados pelo Método System-GMM.

Como, teoricamente o estimador de MQO para a variável dependente defasada proporciona estimativas viesadas para baixo, enquanto os estimadores do Método Within Groups geram estimativas viesadas para cima, uma estimativa adequada para o parâmetro de $\Delta T_{x c \text { escpib }}{ }_{i t-1}$ deve ser compreendida entre os limites desses dois estimadores (MQO e Within Groups).

Descrito os procedimentos econométricos e estatísticos para gerar as estimativas, reserva-se a próxima seção para a análise e discussão dos resultados obtidos pelo Método System-GMM, para que as variâncias dos parâmetros sejam robustas à heterocedasticidade e autocorrelação. Ressaltando que o estimador obtido foi corrigido pelo Método Windmeijer (2005) para evitar que o respectivo estimador subestime as verdadeiras variâncias em amostra finita.

\section{RESULTADOS}

Nessa seção são apresentados os resultados dos três métodos econométricos para dados em painel utilizados para analisarmos o impacto dos gastos públicos em educação e saúde no crescimento econômico dos estados brasileiros.

\subsection{Resultados do modelo efeitos fixos e aleatórios}

A Tabela 2 apresenta os resultados obtidos através da estimativa dos modelos fixos e aleatórios. Deve-se ressaltar que se utilizou a estatística de Hausman, que testa a hipótese dos efeitos de heterogeneidade individual ser ou não correlacionados com os regressores, para testar quais destes modelos é o mais apropriado para cada índice.

De modo que conforme a estatística de Hausman apresentou valor igual a 4,49, indicando aceitar a hipótese nula de que as diferenças entre os parâmetros estimados pelos dois modelos são sistemáticas, aceitando, assim, o modelo com efeitos aleatórios.

Os resultados mostraram que todas as variáveis do modelo são estatisticamente significantes, a nível de 10\% de significância, e com sinal positivo, exceto a taxa de desemprego (LnTXDES). No caso da variável de gastos com educação (Lngastedu), um aumento de $1 \%$ dos gastos em educação em proporção ao PIB proporciona um aumento de $0.05 \%$ na taxa de crescimento econômico dos estados. Já em relação aos gastos com saúde (Lngastsaud), um aumento de $1 \%$ nos gastos com saúde em proporção ao PIB proporciona um aumento de $0.04 \%$ na taxa de crescimento econômico dos estados.

A variável de superávit (ou déficit) primário (SDP) também influencia o crescimento econômico positivamente. Um aumento de $1 \%$ no superávit primário proporciona um aumento de $0,96 \%$ na taxa de crescimento econômico dos estados. Isso indica que quanto mais os estados produzirem superávits fiscais, maiores serão as chances de promover o seu crescimento econômico.

Da mesma maneira, a taxa de câmbio influencia positivamente o crescimento econômico dos estados. Uma desvalorização cambial de $1 \%$ ocasiona um aumento no crescimento econômico dos estados em $0,02 \%$. Isso ocorre devido ao estímulo nas exportações e desestímulo nas importações, o que favorece a balança comercial dos estados.

Por fim, observa-se que a taxa de desemprego influencia negativamente. Ou seja, uma redução de $1 \%$ na taxa de desemprego gera um aumento do crescimento econômico de $0,15 \%$. 
Tabela 2 - Resultados do modelo econométrico de efeitos fixos e aleatórios

\begin{tabular}{|c|c|c|}
\hline Variáveis & Modelo de efeitos fixos & Modelo de efeitos aleatórios+ \\
\hline \multirow{2}{*}{ Lngastedu $_{\mathrm{t}-1}$} & $0.0567^{*}$ & $0.0513^{*}$ \\
\hline & $(0.0160)$ & $(0.0169)$ \\
\hline \multirow{2}{*}{ Lngastsaud $_{t-1}$} & 0.0139 & $0.0435^{\star}$ \\
\hline & $(0.0118)$ & $(0.0219)$ \\
\hline \multirow{2}{*}{$\operatorname{LnSDP}_{\mathrm{t}-1}$} & $2.7545^{\star}$ & $0.9629^{*}$ \\
\hline & $(0.8409)$ & $(0.3821)$ \\
\hline \multirow{2}{*}{$\operatorname{LnTC}_{\mathrm{t}-1}$} & $0.0224^{*}$ & $0.0209^{*}$ \\
\hline & $(0.0072)$ & $(0.0068)$ \\
\hline \multirow{2}{*}{$\operatorname{LnTXDES}_{\mathrm{t}-1}$} & $-0.1996^{*}$ & $-0.1504^{*}$ \\
\hline & $(0.0797)$ & $(0.0571)$ \\
\hline \multirow{4}{*}{ Constante } & -0.2180 & $-0.4331^{*}$ \\
\hline & $(0.1546)$ & $(0.1136)$ \\
\hline & $\mathrm{N}^{\circ}$ de Obs: 594 & Teste de Hausman: 4.49 \\
\hline & No de Grupos: 27 & Prob > chi2: 0.1089 \\
\hline
\end{tabular}

Fonte: Dados da pesquisa. Elaboração dos autores.

OBS: Erro-padrão em parênteses. $\left(^{*}\right)$ representa significância a 10\%. (+) representa o modelo mais apropriado segundo o Teste de Hausman.

\subsection{Resultados do modelo de regressões quantílicas}

A Tabela 2 apresenta os resultados da regressão quantílica para a taxa de crescimento econômico dos estados brasileiros. Vale ressaltar que para a estimação de cada quartil utilizou-se 1.000 replicações bootstrap. Os resultados mostraram que a variável de gastos com educação (Lngastedu) apresentou ser estatisticamente significantes em todos os quantis e apresenta sinal esperado. Ou seja, quanto mais se gasta com educação, maiores são as chances de promover o crescimento econômico dos estados brasileiros. Assim, o quantil que essa variável mais impactou foi o último quantil (0.9). Nesse quantil, o coeficiente referente aos gastos com educação foi de 0,0280 . Ou seja, um aumento de $1 \%$ nos gastos com educação em proporção ao PIB estadual proporciona um aumento de $0.028 \%$ na taxa de crescimento econômico dos estados.

Em relação à variável de gastos com saúde (Lngastsaud), esta só não consegue impactar o crescimento econômico dos estados no quantil 0.25 . Contudo, os gastos com saúde, assim como os gastos com educação, apresentam ter uma relação positiva com o crescimento econômico, visto que em todos os quartis observou-se que os coeficientes apresentaram sinal positivo.

A variável de superávit (ou déficit) primário (SDP) também apresentou sinal positivo, reafirmando que se os estados conseguirem superávits primários, estes poderão promover o crescimento econômico. O maior impacto dessa variável foi quantil 0.10 , com o valor do coeficiente igual a 0,7137 . Ou seja, um aumento do superávit fiscal de $1 \%$ em proporção ao PIB estadual proporciona um aumento de $0,71 \%$ na taxa de crescimento econômico nos estados. A taxa de câmbio mostrou-se significante em todos os quantis, indicando uma resposta positiva no crescimento econômico. Já a taxa de desemprego mostrou-se também significante em todos os quantis, mas apresentando uma resposta negativa. Assim, compreende-se que reduções na taxa de desemprego proporcionam aumentos nas taxas de crescimento econômico. 
Tabela 3 - Resultados das estimativas da regressão quantílica em relação ao crescimento econômico

\begin{tabular}{cccccc}
\hline Quantis & $\mathbf{0 . 1 0}$ & $\mathbf{0 . 2 5}$ & $\mathbf{0 . 5 0}$ & $\mathbf{0 . 7 5}$ & $\mathbf{0 . 9 0}$ \\
\hline \multirow{2}{*}{ Constante } & -0.1257 & 0.0169 & $0.0917^{*}$ & $0.1560^{*}$ & $0.2739^{*}$ \\
& $(0.1557)$ & $(0.0460)$ & $(0.0383)$ & $(0.0525)$ & $(0.1001)$ \\
LnGastedu $_{\mathrm{t}-1}$ & $0.0121^{*}$ & $0.0071^{*}$ & $0.0103^{*}$ & $0.0127^{*}$ & $0.0280^{*}$ \\
& $(0.0067)$ & $(0.0035)$ & $(0.0044)$ & $(0.0062)$ & $(0.0131)$ \\
LnGastsaud $_{\mathrm{t}-1}$ & $0.0249^{*}$ & 0.0039 & $0.0076^{*}$ & $0.0189^{*}$ & $0.0379^{*}$ \\
& $(0.0126)$ & $(0.0076)$ & $(0.00359)$ & $(0.0094)$ & $(0.0152)$ \\
LnSDP $_{\mathrm{t}-1}$ & $0.7137^{*}$ & $0.5306^{*}$ & $0.3867^{*}$ & $0.2914^{* *}$ & $0.1671^{*}$ \\
& $(0.3357)$ & $(0.2521)$ & $(0.2113)$ & $(0.1405)$ & $(0.0712)$ \\
LnTC $_{\mathrm{t}-1}$ & $0.0124^{*}$ & $0.0111^{*}$ & $0.0104^{*}$ & $0.0091^{*}$ & $0.0077^{*}$ \\
& $(0.0041)$ & $(0.0018)$ & $(0.0017)$ & $(0.0016)$ & $(0.0041)$ \\
LnTXDES $_{\mathrm{t}-1}$ & $-0.0479^{*}$ & $-0.0409^{*}$ & $-0.0398^{*}$ & $-0.0306^{* *}$ & $-0.0697^{*}$ \\
Pseudo R $^{2}$ & $(0.0214)$ & $(0.0117)$ & $(0.0104)$ & $(0.0135)$ & $(0.0260)$ \\
\hline
\end{tabular}

Fonte: Dados da pesquisa.

OBS: Erro-padrão em parênteses. ( ${ }^{*}$ ) representa significância a 10\%.

\subsection{Resultados do modelo GMM}

A Tabela 4 apresenta os resultados do modelo dos Momentos Generalizados (GMM) visando verificar o impacto dos gastos público no crescimento econômico dos estados brasileiros.

Inicialmente, estimou-se a equação 8 por MQO e pelo Método Within Groups $\ln \left(R m_{i, t}\right)$. Para selecionar o estimado, consideraram-se os resultados dos testes de especificação de Hausman para verificar se Lngastedu, Lngastsaud, LnSDP, LnTC e LnTXDES são endógenas, além da verificação da validade dos instrumentos por meio dos Testes de Hansen e Sargan. De acordo com os resultados obtidos pelos testes, não é necessário tratar essas variáveis como endógenas e, ainda, da utilização das segundas defasagens das variáveis e os instrumentos adicionais requeridos pelo System-GMM serem tratados como válidos.

Além destes testes, foram realizados testes de captar a presença de autocorrelação de primeira ordem dos resíduos em nível e em primeira diferença (isto é, segunda ordem). Observou-se que não se rejeita a hipótese nula, ou seja, os resíduos apresentam correlação de primeira ordem. Assim, para que as estimativas não sejam viesadas, é necessário mitigar a presença desta e um dos mecanismos para isto é estimar o modelo em primeira diferença como, por exemplo, por meio do estimador System-GMM.

Diante disso, considera-se para efeito de análise dos resultados as estimativas obtidas pelo System-GMM. Ressalte-se que a apresentação das estimativas por MQO e Within Group se dá unicamente para mostrar que o valor estimado da variável dependente defasada se encontra, respectivamente, entre os valores estimados obtidos por esses dois métodos, servindo como um possível indicador de que o viés causado pela presença de variáveis endógenas e de efeitos fixos não observáveis é corrigido pelo Método System-GMM.

Segundo os resultados, a hipótese de persistência do crescimento econômico dos estados brasileiros parece se confirmar ao se verificar a significância estatística da variável $T x_{c r e s c p i b}{ }_{i t-1}(0.2136)$. Esse resultado indica que a taxa de crescimento econômico dos estados é influenciada pelo seu comportamento no passado.

As variáveis: gastos com educação (Lngastedu), gastos com saúde (Lngastsaud), superávit (ou déficit) primário (SDP) e taxa de câmbio (LnTC) apresentaram-se como estatisticamente significantes e com sinal positivo. Ou seja, impactam positivamente a taxa de crescimento econômico dos estados brasileiros. Já a variável taxa de desemprego apresentou um sinal negativo, impactando de forma negativa o crescimento econômico dos estados brasileiros.

A variável de gastos com educação (Lngastedu) apresentou um coeficiente igual a 0.0271 , indicando que um aumento de $1 \%$ nos gastos dos governos estaduais com educação, em proporção do seu PIB, proporciona um aumento na taxa de crescimento econômico de $0.027 \%$. Já a variável gastos com saúde (Lngastsaud) apresentou um coeficiente igual a 0.0177 , indicando que um aumento nos gastos com saúde em proporção ao seu PIB ocasiona um aumento na taxa de crescimento econômico de $0.017 \%$.

A variável de superávit (ou déficit) primário (SDP) apresentou ter uma relação positiva com a taxa de crescimento econômico dos estados. Isso implica dizer que quanto maior for o superávit fiscal alcançado pelos governos estaduais, maior será o crescimento econômico dos estados brasileiros. O coeficiente igual a 0,9583 indica que um aumento no superávit fiscal de $1 \%$ proporciona um aumento na taxa de crescimento econômico dos estados de $0,95 \%$. 
A taxa de câmbio apresentou um coeficiente igual a 0,0243. Assim, uma desvalorização cambial de $1 \%$ ocasiona um aumento de $0,02 \%$ na taxa de crescimento econômico dos estados brasileiros. Já a taxa de desemprego, com o coeficiente igual a -0,1095 indica que uma redução na taxa de desemprego de $1 \%$ gera um aumento da taxa de crescimento econômico nos estados de 0,1095\%.

Tabela 4 - Resultados do modelo GMM

\begin{tabular}{|c|c|c|c|}
\hline \multirow{2}{*}{ Variáveis explicativas } & \multicolumn{3}{|c|}{ Txcrescpib } \\
\hline & MQO & Within groups & GMM- System \\
\hline \multirow{2}{*}{$T_{x c r e s c p i b}{ }_{i t-1}$} & $0,2047^{*}$ & $0.2812^{*}$ & $0.2136^{*}$ \\
\hline & $(0,0423)$ & $(0,0419)$ & $(0.0116)$ \\
\hline \multirow{2}{*}{ Lngasted $u_{i, t-1}$} & $0.0253^{*}$ & $0.0284^{*}$ & $0.0271^{*}$ \\
\hline & $(0,0151)$ & $(0.0127)$ & $(0.0121)$ \\
\hline \multirow{2}{*}{ Lngastsaud $_{i, t-1}$} & $0.0545^{\star \star}$ & $0.0179^{*}$ & $0.0177^{*}$ \\
\hline & $(0,0273)$ & $(0.0081)$ & $(0.0082)$ \\
\hline \multirow{2}{*}{$\operatorname{LnSDP_{i,t-1}}$} & $0.6012^{*}$ & $1.4637^{*}$ & $0.9583^{*}$ \\
\hline & $(0.1815)$ & $(0.6753)$ & $(0.4407)$ \\
\hline \multirow{2}{*}{$\operatorname{LnTC}_{i, t-1}$} & $0.0226^{*}$ & $0.0253^{*}$ & $0.0243^{*}$ \\
\hline & $(0.0067)$ & $(0.0069)$ & $(0.0114)$ \\
\hline \multirow{2}{*}{$\operatorname{LnTXDES}_{i, t-1}$} & $-0.0747^{* *}$ & $-0.1178^{*}$ & $-0.1095^{\star}$ \\
\hline & $(0.0353)$ & $(0.0566)$ & $(0.0522)$ \\
\hline \multirow{2}{*}{ Const. } & $-0.5477^{*}$ & $-0.3957^{*}$ & $-1.1708^{*}$ \\
\hline & $(0,1106)$ & $(0.2266))$ & $(0.0295)$ \\
\hline Estatística F & 13.75 & 10.21 & 22.19 \\
\hline P-valor & 0,0000 & 0,0000 & 0,0000 \\
\hline $\mathbf{R}^{2}$ & 0,0817 & - & - \\
\hline $\mathbf{N}$ & 567 & 567 & 567 \\
\hline Grupos & 27 & 27 & 27 \\
\hline \multicolumn{2}{|c|}{$\mathrm{H}_{0}$ : Ausência de autocorrelação nos resíduos de primeira ordem } & P-valor & 0,027 \\
\hline \multicolumn{2}{|c|}{$\mathrm{H}_{0}$ : Ausência de autocorrelação nos resíduos de segunda ordem } & P-valor & 0,236 \\
\hline \multicolumn{2}{|c|}{ Teste de Hansen } & Prob>chi2 & 0,253 \\
\hline \multicolumn{2}{|c|}{ Teste de Sargan } & Prob>chi2 & 0,495 \\
\hline
\end{tabular}

Fonte: Elaboração dos autores utilizando dados da pesquisa.

OBS: Erro-padrão em parênteses. $\left({ }^{*}\right)$ representa significância a $10 \%$.

Assim, conclui-se que, de uma forma geral, há robustez nos resultados, independentemente do método utilizado, e que o aparente impacto dos gastos públicos em educação e saúde, no crescimento econômico dos estados brasileiros, segue aquilo encontrado tanto na literatura internacional, quanto nos estudos voltados para o Brasil.

Observa-se, por exemplo, que os resultados estão de acordo com os encontrados em Santos (2008), que encontra relação positiva entre gastos em saúde e anos de estudo com o crescimento econômico dos municípios cearenses. Além disso, também é argumentado, em Tarda e Rodrigues (2015), que se encontra relação positiva entre os gastos em educação e o crescimento econômico no contexto específico da cidade de Campinas, São Paulo, ou, ainda, na relação encontrada por Vagt, Degenhart e Zonatto (2015), dos gastos públicos com educação e saúde, que motivam impacto no crescimento para os municípios da região sudeste do Brasil.

Da mesma maneira, os resultados corroboram o encontrado em diversas contribuições internacionais, como, por exemplo, Campodonico, Cassinelli e Mesones (2014), que encontram impacto positivo de gastos em educação e saúde (além de infraestrutura) no crescimento econômico do Peru, assim como também o encontrado por Lacheheb, Nor e Baloch (2014), que sugerem que a relação entre gastos em educação e saúde e crescimento econômico, nos países do Oriente Médio e África da amostra, é positiva. Os resultados também estão alinhados, pelo menos no quesito educação, pelo robusto estudo feito por Churchill, Yew e Ugur (2015), de extensa amostra de período e países (membros da OCDE), que também verifica efeito positivo entre gastos em educação e crescimento econômico, ainda que a relação entre gastos em saúde e crescimento, naquele estudo, tenha se mostrado insignificante. 


\section{CONCLUSÕES}

O presente estudo teve como objetivo analisar o impacto dos gastos públicos no crescimento econômico dos estados brasileiros. Para isso, utilizaram-se um painel de dados de gastos com educação, saúde, superávit (ou déficit) primários, taxa de câmbio e taxa de desemprego; além da taxa de crescimento real do Produto Interno Brutos das 27 unidades federativas do Brasil, compreendendo o período de 1991 a 2012. O período escolhido deu-se com base na disponibilidade de dados, assim como a intenção de adequá-lo ao período mencionado por parte considerável da literatura revisada na segunda seção do trabalho.

Além disso, utilizaram-se três modelos econométricos visando captar esse impacto: método de efeitos fixos e aleatórios, regressões quantílicas e Método dos Momentos Generalizados (GMM) desenvolvido por Blundell-Bond (1998). Assim, compreende-se que todos os métodos são para dados em painel.

Os resultados sugerem que, para todas as três metodologias utilizadas no estudo, tanto os gastos com educação quanto os gastos com saúde impactam, mesmo que de forma tímida, positivamente no crescimento econômico, corroborando com os estudos desenvolvidos por Baldacci et al. (2008), Santos (2008), Kambiz Peykarjouet et al. (2011), Churchill, Yew e Ugur (2015), Campodonico, Cassinelli e Mesones (2014) Lacheheb, Nor e Baloch (2014), Silva (2012), Tarda e Rodrigues (2015) e Vagt, Degenhart e Zonatto (2015).

Outra variável que impactou positivamente o crescimento econômico dos estados brasileiros foi a adoção de política fiscal ortodoxa, que leva a superávits fiscais. Em todas as metodologias analisadas, essa variável se mostrou bastante relevante na geração do crescimento econômico. Além disso, desvalorizações cambiais influenciam positivamente na geração de superávits na balança comercial, independentemente do método utilizado. Ou seja, a intuição seria que os estados que conseguem manter suas metas fiscais e utilizam seus superávits, tanto fiscais quanto comerciais, têm maiores condições de investimentos em infraestrutura, pagamento da dívida pública e de atrair novos investimentos na região, motivando assim o crescimento econômico.

A grosso modo, portanto, é possível sugerir que o gasto público canalizado para iniciativas em educação e saúde é interessante para motivar o crescimento econômico, como observado na literatura acadêmica sobre o tema. Especificamente para o contexto brasileiro abordado no presente trabalho, na observação de décadas com crescimento econômico carente de robustez, e com perenes problemas nas duas searas, tais iniciativas seriam de particular importância. No entanto, os resultados sugerem que esse gasto deve ser feito de maneira eficiente, pois a higidez fiscal mostra-se também relevante para o crescimento econômico, i.e., além da análise cautelosa com o gasto desmesurado.

Ademais, pode-se concluir que, por mais que as variáveis acima mencionadas sejam importantes na geração do crescimento econômico, novos estudos devem buscar verificar também outros fatores que promovam tal crescimento. Isso fica evidente quando verificamos, por exemplo, os pseudos $\mathrm{R}^{2}$ das regressões quantílicas, que apesar de serem significantes, apresentam baixos valores, gerando indícios que outras variáveis podem explicar o crescimento econômico nos estados brasileiros.

\section{REFERÊNCIAS}

ABU-BADER, S.; ABU-QARN, A. S. Government expenditu-res, military spending, and economic growth: causality evidence from Egypt, Israel, and Syria. Journal of Policy Modeling, [S. I.], v. 25, n. 6, p. 567-583, 2003.

AHN, S. C.; SCHIMDT, P. Efficient estimation of models for dynamic panel data. Journal of Econometrics, [S. I.], v. 68, p. $5-28,1995$.

ALSHAHRANI, S. A.; ALSADIQ, A. J. Economic growth and government spending in Saudi Arabia: an empirical investigation. Washington: IMF Working Paper. 2014.

ARAÚJO, J. A.; MONTEIRO, V. B.; MORAIS, G. S. Gastos públicos e crescimento econômico: evidências da economia cearense. In: ENCONTRO - ECONOMIA DO CEARÁ EM DEBATE, 8., 2012, Fortaleza. Anais [...]. Fortaleza: IPECE, 2012, 20p. Disponível em: Link. Acesso em: 22 jun. 2018.

ARELLANO, M.; BOND, S. Some tests of specification for panel data: Monte Carlo evidence and an application to employment equations. The Review of Economic Studies, [S. I.], v. 58, n. 2, p. 277-297, 1991.

ARELLANO, M.; BOVER, O. Another look at the instrumental-variable estimation of error components model. Journal of Econometrics, [S. I.], v. 68, p. 29-52, 1995.

ASCHAUER, D. Is public expenditure productive? Journal of Monetary Economics, [S. I.], v. 23, p. 177-200,1989. 
AURANGZEB. Relationship between health expenditure and GDP in an augmented solow growth model for Pakistan: an application of co-integration and error-correction modeling. Lahore Journal of Economics, [S. I.], v. 8, n. 2, p. 1-16, Jul./Dec. 2003.

AYUBA, A. J. The relationship between public social expenditure and economic growth in Nigeria: an empirical analysis. International Journal of Finance and Accounting, [S. I.], v. 3, n. 3, p. 185-191, 2014.

BAKARE A. S.; OLUBOKUN, Sanmi, Health care expenditure and economic growth in Nigeria: an empirical study. Journal of Emerging Trends in Economics and Management Sciences, [S. I.], v. 2, n. 2, p. 83-87. 2011.

BALDACCI, E.; CLEMENTS, B.; GUPTA, S.; CUI, Q. Social spending, human capital, and growth in developing countries. World Development, [S. I.], v. 36, n. 8, p. 1317-1341, 2008.

BARRO, R. J. Government spending in a simple model of endogenous growth. Journal of Political Economy, [S. I.], v. 98, p. 103-125, 1990.

BARRO, R. J. Economic growth in a cross-section of countries. The Quarterly Journal of Economics, [S. I.], v. 106, p. 407-444, 1991.

BARBOSA, W. F. ; SOUSA, E. P. Eficiência técnica e de escala do Sistema Único de Saúde nos municípios do Nordeste brasileiro. Revista Econômica do Nordeste, [S. I.], v. 46, p. 99-113, 2015.

BARRO, R. J.; SALA-I-MARTIN, X. Economic Growth. Nova York: McGraw, 1995.

BEDIR, S. Healthcare expenditure and economic growth in developing countries. Advances in Economics and Business, [S. I.], v. 4, n. 2, p. 76-86, 2016.

BEZERRA, E. P.; DIWAN, J. R. Uso de DEA como alternativa ao IDH na mensuração do desenvolvimento humano nos maiores municípios brasileiros. Trabalho de Conclusão de Curso. (Bacharelado em Engenharia de Produção) - Universidade Federal do Rio de Janeiro, Rio de Janeiro, 2001.

BLUNDELL, R.; BOND, S. Initial conditions and moment restrictions in dynamic panel data models. Journal of Econometrics, [S. I.], v. 87, p. 115-143, 1998.

BOGONI, N. M.; BEUREN, I. M.; HEIN, N. Análise da relação entre crescimento econômico e gastos públicos nas maiores cidades da região Sul do Brasil. Revista de Administração Pública, Rio de Janeiro, v. 45, n. 1, p. 159-179, jan./fev. 2011.

BLOOM, D. E.; CANNING, D.; SEVILLA, J. The effect of health on economic growth: a production function approach. World Development, [S. I.], v. 32, n. 1, p. 1-13, 2004.

BOUSSALEM, F.; BOUSSALEN, Z.; TAIBA, A. The relationship between public spending on health and economic growth in algeria: testing for co-integration and causality. International Journal of Business and Management, [S. I.], v. 2, n. 3, p. 25-39, 2014.

BRUNET, J. F. G.; BORGES, C. B.; BERTÊ, A. M. A.; BUSSATO, L. M. Estados comparados por funções do orçamento - uma avaliação da eficiência e efetividade dos gastos públicos estaduais. Porto Alegre: [s. n.], 2006.

BUCHNANAN, J. Rent-seeking and profit-seeking. In: Tollison, Robert D.; TULLOCKS, Gordon (ed.). Toward a theory of the rent-seeking society. Texas: Texas A\&M University Press. 1980. p. 3-15.

CAMPODONICO, J. F. B.; CASSINELLI, J. R. P.; MESONES, J. A. The impact of public expenditures in education, health, and infrastructure on economic growth and income distribution in Peru. Lima: PredicePeru, 2014. (IDB Working Paper Series, 490).

CÂNDIDO JR., J. Os gastos públicos no Brasil são produtivos? Brasília: IPEA, 2001. (Texto para Discussão, 781).

CHURCHILL, S. A.; YEW, S. L.; UGUR, M. Effects of government education and health expenditures on economic growth: a meta-analysis. [S. I.]: GPERC21, 2015. 
COLE, M.; NEYMAYER, E. The impact of poor health on total factor productivity. Journal of Development Studies, [S. I.], v. 42, n. 6, p. 918-938. 2006.

DEVERAJAN, S.; SWAROOP, V.; ZOU, H.-F. The composition of public expenditure and economic growth. Journal of Monetary Economics, [S. I.], v. 37, n. 2, p. 313-344,1996. DEVLIN, N.; HANSEN, P. Health care spending and economic output: granger causality. applied economics letters, [S. I.], v. 8, p. 561-564, 2001.

EGGOH, J.; HOUEINVO, H.; SOSSOU, G. A. Education, health and economic growth in African countries. Journal of Economic Development, [S. I.], v. 40, n. 1. p. 93-111, 2015.

FARIA, F. P. Gastos sociais e condições de vida nos municípios fluminenses: uma avaliação através da análise envoltória de dados. 2005. Dissertação (Mestrado em Estudos Populacionais e Pesquisas Sociais) - Escola Nacional de Ciências Estatísticas, Rio de Janeiro, 2005.

GERARDO, J. C. Política fiscal e sustentabilidade do crescimento. [S. I.: s. n.], 2010.

GLOMM, G.; RAVIKUMAR, B. Public versus private investment in human capital: endogenous growth and income inequality. The Journal of Political Economy, [S. I.], v. 100, n. 4 p. 818-834, 1992.

GREENE, W. H. Econometric analysis. New Jersey: Prentice Hall, 2000.

GROSSMAN, M. On the concept of health capital and the demand for health. Journal of Political Economy, [S. I.], v. 80 , n. 2, p. 223-255.1972.

KAMBIZ, PEYKARJOU et al. Studying the relationship between health and economic growth in OIC member states. Interdisciplinary Journal of Contemporary Research in Business. v. 3, n. 8. p. 1041-1054, 2011.

KEYNES, J. M. The general theory of employment, interest and money. United Kingdom: Palgrave McMillan, 1936.

LACHEHEB, M.; MED NOR, N.; BALOCH, I. Health expenditure, education and economic growth in MENA countries. Germany: University Library of Munich, 2014.

LILLARD, L.; WEISS, Y. Uncertain health and survival: effects on end-of-life consumption. Journal of Business \& Economic Statistics, [S. I.], v. 15, n. 2, p. 254-68, 1997.

LUSTIG, N. Investing in health for economic development: the case of Mexico. Finland: World Institute for Development Economic Research, 2006.

MAITA, B.; MUKHOPADHYAY. C. K. Public spending on education, health care and economic growth in selected countries of Asia and the Pacific. Asia-Pacific Development Journal, [S. I.], v. 19, n. 2, p. 19-48, Dec. 2012.

MARINHO, A. Avaliação da eficiência técnica nos serviços de saúde dos municípios do estado do Rio de Janeiro. Rio de Janeiro: IPEA, 2001. (Texto para discussão, 842).

MARQUES JR, L. S.; OLIVEIRA, C. A.; JACINTO, P. da A. O papel da política fiscal local no crescimento econômico de cidades: uma evidência empírica para o Brasil. In: ENCONTRO NACIONAL DE ECONOMIA, 34., 2006. Salvador. Anais [...]. Salvador: ANPEC, 2006. p. 1-20.

OLIVEIRA, C. A. de; LIMA, F. S. de. Impacto do crescimento, da concentração de renda e das transferências governamentais sobre a pobreza nos municípios brasileiros. Análise Econômica. Porto Alegre, ano 28, n. 53, p. 159-180, 2010.

ONI, L. B. Analysis of the growth impact of health expenditure in Nigeria. Journal of Economics and Finance, [S. I.], v. 3, n. 1, p. 77-84, fev. 2014.

PENNA, C. M.; TROMPIERI NETO, N. Renda do trabalho, renda das transferências e desigualdade: uma nova proposta de investigação empírica da Curva de Kuznets para o Ceará. Fortaleza: IPECE, 2009. (Texto Para Discussão, 66.). 
PIRIM, Zafer, OWINGS, Leslie S. KAPLAN. The long-term impact of educational and health spending on unemployment rates. European Journal of Economic and Political Studies, [S. I.], v. 7, p. 49-69, 2014.

RAM, R. Causality between Income and government expenditure: a broad international perspective, Public Finance, [S. I.], v. 3, p. 393-13, 1986.

ROCHA, F.; GIUBERT, A. C. Composição do gasto público e crescimento econômico: uma avaliação macroeconômica da qualidade dos gastos dos Estados brasileiros. Econ. Aplic., São Paulo, v. 11, n. 4, p. 463-485, out./dez. 2007.

RODRIGUES, R. V.; TEIXEIRA, E. C. Gasto púbico e crescimento econômico no Brasil: uma análise comparativa dos gastos das esferas do governo. Revista Brasileira de Economia, [S. I.], v. 64, n. 4, p. 423-438, 2010.

SANT'ANNA, J. M. B. Efeito do gasto público sobre o PIB: um teste empírico nos Municípios do Estado do Espírito Santo. 2006. Dissertação (Mestrado em Ciências Contábeis) - Fundação Instituto Capixaba de Pesquisas em Contabilidade, Economia e Finanças, Vitória: FUCAPE, 2006.

SANTOS, R. C. L. O efeito do gasto público sobre o PIB dos municípios cearenses. Dissertação (Mestrado em Economia) - Universidade Federal do Ceará, Fortaleza: UFC, 2008.

SILVA, L. M. C. A relação entre os gastos públicos e o crescimento econômico: uma análise para os municípios paraibanos no período 2000-2008. 2012. Dissertação (Mestrado em Economia) - Universidade Federal da Paraíba, João Pessoa: UFPB, 2012.

SOLOW, R. A contribution to the theory of economic growth. Quarterly Journal of Economics, [S. I.], v. 70, n. 1, p. 65-94, 1956.

SRINIVASAN, THIRUKODIKAVAL N. Neoclassical political economy, the state and economic development. Asian Development Review, v. 3, n. 2, p. 38-58, 1985.

SUMMERS, R.; HESTON, A. A new set of international comparisons of real product and price levels. Estimates for 130 countries. Review of Income and Wealth, [S. I.], v. 34, p. 1-25, 1988.

SUHRCKE, M.; VÖRK, A.; MAZZUCO, S. The economic consequences of ill-health in Estonia. Talín: World Health Organization, 2006.

TARDA, K. B.; RODRIGUES, R. V. A influência dos gastos em educação no crescimento econômico da região administrativa de campinas. ESTUDO \& DEBATE, Lajeado, v. 22, n. 2, p. 7-31, 2015.

VENTELOU, Bruno; BRY, Xavier. The role of public spending in economic growth: envelopment methods. Journal of Policy Modeling, [S. I.], v. 28, n. 4, p. 403-413. 2006.

VOGT, M.; DESENHART, L.; ZONATTO, V. C. da S. Relação entre o crescimento econômico e os gastos públicos em municípios da região Sudeste do Brasil. CONGRESSO ANPCONT, 9., 2015, Curitiba. Anais [...]. Curitiba: ANPCONT, 2015. p. 1-19.

WINDMEIJER, F. A finite sample correction for the variance of linear efficient two-step GMM estimators. Journal of Econometrics, [S. I.], v. 126, n. 1, p. 25-51, 2005. 


\section{Contato:}

Francisco José Silva Tabosa

E-mail: franzetabosa@ufc.br

Pablo Urano de Carvalho Castelar

E-mail: pcastelar@ufc.br 